\title{
Improving the performance of three level code division multiplexing using the optimization of signal level spacing
}

\begin{abstract}
In order to optimize the performance of three level code division multiplexing (3LCDM) at $2 \times 20 \mathrm{~Gb} / \mathrm{s}$ data rate, signal level spacing technique is investigated in this paper. The $3 \mathrm{LCDM}$ performance is improved considerably using both electrical and optical level spacing optimization configurations. The results demonstrate that by optimization, in conditions of the optical signal-to-noise ratio, an improvement of around $4.5 \mathrm{~dB}$ can be achieved in both approaches as well as $3.3 \mathrm{~dB}$ in the electrical configuration and $3.5 \mathrm{~dB}$ in the optical configuration can be accomplished for the $3 \mathrm{LCDM}$ in terms of the receiver sensitivity.
\end{abstract}

Keyword: Optical communication; 3LCDM; Dual drive mach zehnder; Multiplexing; Level spacing 